\title{
Research on High Speed Mobile Payment Technology Based on License Plate Recognition
}

\author{
Ligeng Qin ${ }^{\mathrm{a}}$, Hua Pan ${ }^{\mathrm{b}}$ and Yuzhuo Huang ${ }^{\mathrm{c}}$
}

\author{
Guangxi transportation research \& consulting co., Itd, China. \\ a46557560@qq.com, b597310014@qq.com, c15677180307@163.com
}

\begin{abstract}
Aiming at the demand of high-speed mobile payment, based on the analysis of existing license plate recognition technology, a mobile payment scheme based on license plate recognition is provided. The scheme takes license plate recognition as the core and relies on the Internet, cloud computing and big data to realize fast transaction methods such as no parking, no card and no cash. The program coexists with the existing ETC and MTC charging methods. The real-time network verification with the public security vehicle management database will support the reality of the user's vehicle information. Finally, the feasibility and effectiveness of the scheme are verified by simulating the "license plate recognition + mobile payment" method on the Guangxi Expressway.
\end{abstract}

Keywords: License Plate Recognition, Highway, Mobile Payment.

\section{Introduction}

As a non-cash payment method, ETC technology improves vehicle traffic efficiency, reduces cash management costs, and makes capital recharge and settlement methods more flexible. At the same time, it was quickly promoted through cooperation with banks. However, the handling and initial installation of ETC is relatively complicated, requiring users, vehicles, and personnel to be present at the same time, which also restricts the development of ETC users to a certain extent. Therefore, how to adapt to the development trend and apply new ideas to realize the speed-up, cost reduction and management efficiency of toll stations has become the main problem we have to solve.

This paper studies the mobile phone mobile payment technology based on license plate recognition. After more than ten years of development, the license plate recognition technology has matured and can meet the needs of vehicle traffic identification. The real-time network verification with the public security vehicle management database will support the reality of user vehicle information. Mobile payment and mobile applications can fully meet user needs. In addition, the network security solution can support the operation of mobile payment charging services, and the subsequent big data and account funds will provide a solid foundation for the new industrial layout of Guangxi Expressway.

At present, similar projects at home and abroad have adopted or will soon adopt the card identification method combined with mobile payment means to solve the demand for highway tolls. In the US, I-405 Express Lanes has adopted license plate recognition as a means of charging. In Fujian, China, its expressway service organization has been researching and formulating a charging scheme that uses "license plate recognition + mobile payment" methods.

Regardless of how the future charging method develops, the final direction will be to use the electronic license plate as the only identification factor. Therefore, this paper will work hard in the construction of lane recognition facilities, real-time verification of public security vehicle management data, big data application platform, mobile payment platform, network bearer support and security upgrade. At the same time, all of the above-mentioned investments and all-round highspeed service mobile applications will be used in the future development of the integration with the electronic license plate recognition method. It also pre-arranged the development of the national highway "13th Five-Year Plan" and the transformation of user service Internet.

\section{Literature Review}

Qiu et al. [1] proposed a highway toll collection system scheme, which uses license plate recognition assistance to obtain vehicle information by manual identification, and pays for highways 
through mobile payment, providing a new idea for the highway to achieve the development goal of non-stop charging.

Liu Binbin's [2] research on the workflow of license plate recognition technology equipment and the specific application in high-speed toll shows that the automatic license plate recognition system in the highway toll collection process reduces the workload of the staff and improves the traffic volume of the expressway. It has reference significance for this study.

Zhang Bo [3] proposed the highway-based mobile phone payment platform based on the Internet of Things, which relieves the pressure on the operation and maintenance of the highway from another aspect and enhances the user experience.

Starting from the concept and characteristics of mobile payment, Wang Qiulan [4] discusses its application design and basic composition in the highway toll collection system, and provides theoretical support for realizing mobile payment in the highway toll collection system.

Ding Qifeng [5] discussed the advantages and disadvantages of several representative toll mobile payment schemes in China, and provided reference for the highway decision-making department.

Luo Qingyi et al. [6] combined with the current situation of mobile payment construction in China's highway toll collection system, studied the application of mobile payment in the highway toll field from different dimensions, and compared and evaluated each mobile payment form. The study provides technical decision-making for high-speed road management and management.

Wang Guogang et al. [7] discussed the groundbreaking "Intelligent Redundant License Plate Recognition Technology", which provides a powerful back-office guarantee for highway inspection and evacuation.

Cao Xiaofeng [8] discussed the highway non-stop mobile payment system from the aspects of key technologies, system characteristics and application promotion. From the perspective of the highway operation manager, he explores how to cope with the new challenges of highway toll collection under the situation that "new Internet + technologies" are constantly emerging and charging scenarios are constantly enriched.

Huang Weizhong [9] proposed that the ETC system should introduce "Internet + " thinking, continue to innovate, improve and optimize in terms of service, payment and application, and provide guidance for the research of this paper.

In terms of application fields, Cui Hongjun et al. [10] have designed the new expressway non-stop charging comprehensive control system based on the network payment platform, which is conducive to speeding up the process of highway toll collection and the sustainable development of expressways.

\section{Research Needs}

At present, there are two types of highway tolls:

\subsection{Manual Toll Collection Based on IC Card (MTC).}

The basic process of artificial semi-automation is "manual judgment / automatic judgment, entrance manual card issuing (or self-development card), camera capture license plate number, export inspection card, inspection license plate, calculation toll, manual charge, computer management, video insight, detector check".

As a cash payment method, MTC requires a large number of toll collectors to issue and recycle IC cards, resulting in high labor costs and slower vehicle traffic. The work of checking cash also increases management costs.

\subsection{Electronic Toll Collection Based on RFID Technology (ETC).}

The basic process of the non-stop ETC is that the ETC roadside unit antenna RSU communicates with the onboard unit OBU information to obtain information such as the model, entrance, and time, and realizes fast charging.

As a non-cash payment method, ETC improves vehicle traffic efficiency, reduces cash management costs, and makes capital recharge and settlement methods more flexible. It was quickly 
promoted through cooperation with banks. However, the handling and initial installation of ETC is relatively complicated, requiring users, vehicles, and personnel to be present at the same time.

With the development of mobile Internet and electronic technology, non-cash payment applications have become very extensive and will become an irresistible trend. Therefore, the existing highway toll collection methods need to be promoted the technology upgrades simultaneously.

This paper satisfies the needs of "Internet + highway" and meets the need to accelerate the in-depth integration and innovation of the Internet and Guangxi highway toll collection. Users, vehicles, and road managers are connected through the Internet to achieve self-service application, automatic license plate recognition, no parking, system automatic charge, and manual/automatic acceptance of road conditions.

The specific needs are as follows:

(1) Improve management efficiency and reduce management costs.

With the development of the economy and the surge in vehicle ownership, the traffic volume and usage rate of expressways have increased year by year. At the same time, the improvement of expressway service quality is also under pressure. Purely increasing personnel can no longer meet the needs of toll collection and road management. We should make great efforts to improve management efficiency, reduce management costs, and improve the level of informatization. The implementation of this paper can achieve non-cash payment and non-stop charging, reduce the input of the toll collectors, reduce the risk of cash collection and cash management, thereby improving the efficiency of highway management and reducing management costs.

(2) Improve toll station efficiency.

The implementation of this paper can realize the mobile phone payment without parking, which is half the transit time of the manual toll lane, thus effectively improving the traffic efficiency of the toll station and reducing the congestion of the toll station.

(3) Provide users with a variety of convenient non-cash payment methods.

Compared with ETC, which needs to install on-board unit and bank on-site signing, this article uses mobile phone app to realize user self-service online registration to save users' time, avoiding the inconvenience of on-board installation and banking service of on-board equipment, providing users with more convenient non-cash payment methods outside ETC, leaving convenience to users. The system also provides front-end technical conditions for future electronic license plate recognition payments, ensuring seamless docking.

(4) Expressway intelligent induction and big data analysis.

The system can push the road condition information to the user's app, and the app can voice broadcast road condition information and prompt according to the user's location in advance. The system also obtains user information, travel habits, vehicle location information, etc. as a data source for highway big data construction, providing the basis for big data analysis and mining for road safety management, emergency intelligence, emergency dispatch, intelligent induction, and decision support.

\section{Mobile Payment Scheme Based on License Plate Recognition}

The overall architecture of the system is divided as follows: 


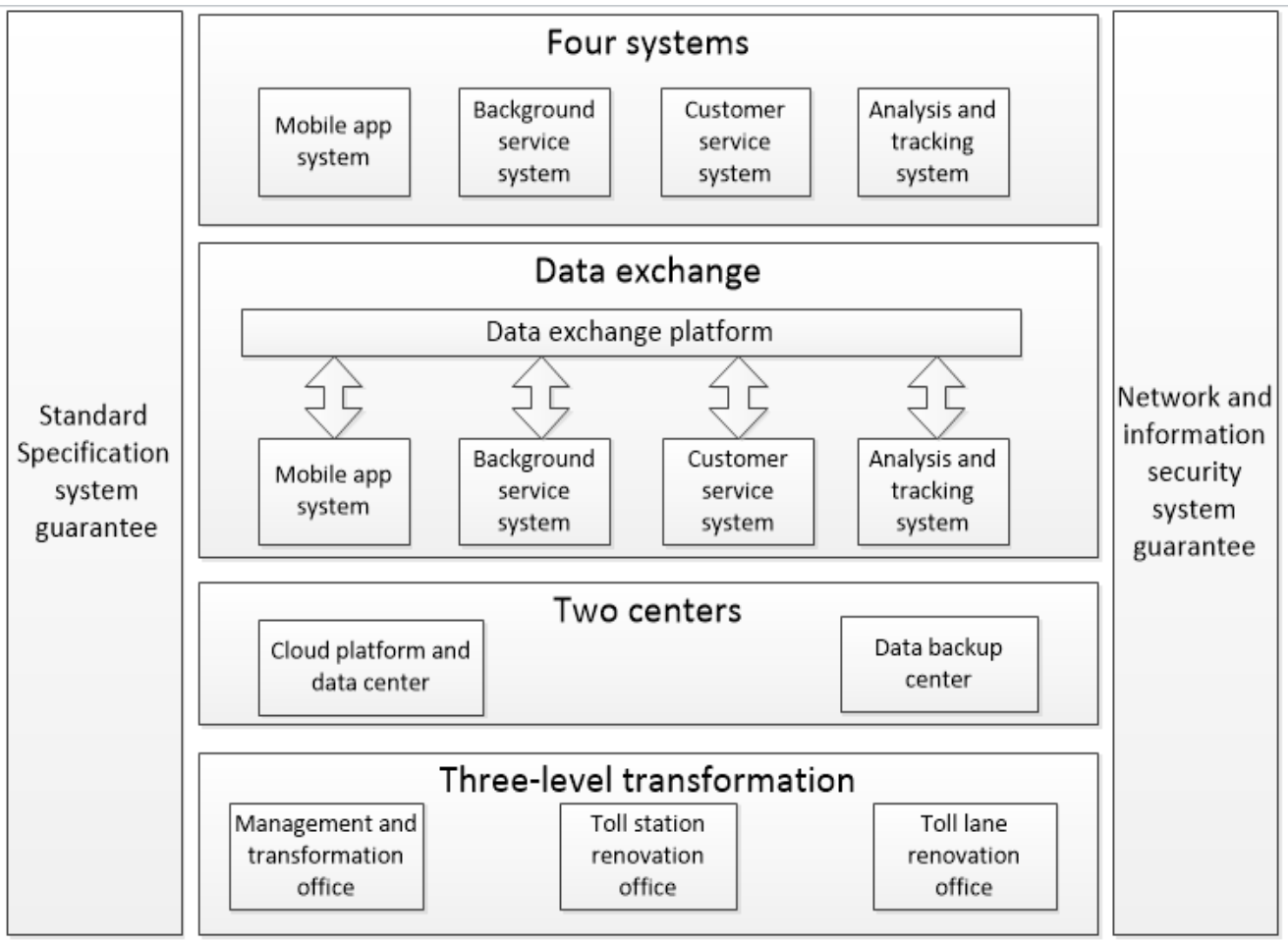

Fig. 1 System architecture

Based on the user-oriented concept, the system adopts the model of "license plate recognition + mobile APP", which can achieve the goal of self-service application for non-stop charging in ETC lane and non-cash payment in MTC lane without additional equipment. The payment scheme is a new charging method other than the existing ETC and MTC charging methods, but coexists with the existing charging methods, improving the overall traffic efficiency, increasing the non-cash payment path for users, and reducing the cost of highway toll management.

The average time for MTC lane entry is $8 \mathrm{~s}$, and the average export time is $16 \mathrm{~s}$. After using the vehicle data dynamic distribution technology, the MTC lane entrance and exit takes an average of 3 to 5 seconds, and the traffic efficiency is doubled.

Flow chart of "License Plate Recognition + Mobile APP" model:
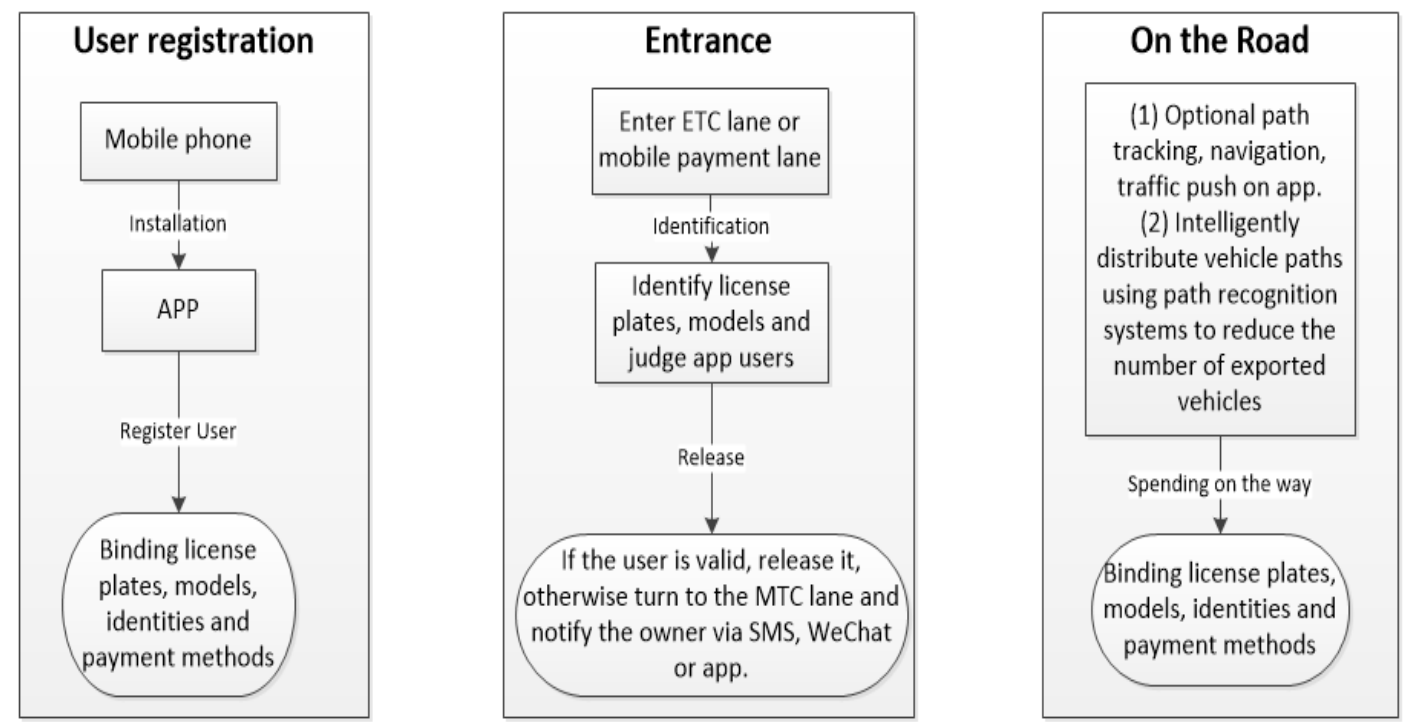

Fig. 2 Flow chart of "License Plate Recognition + Mobile APP” model 


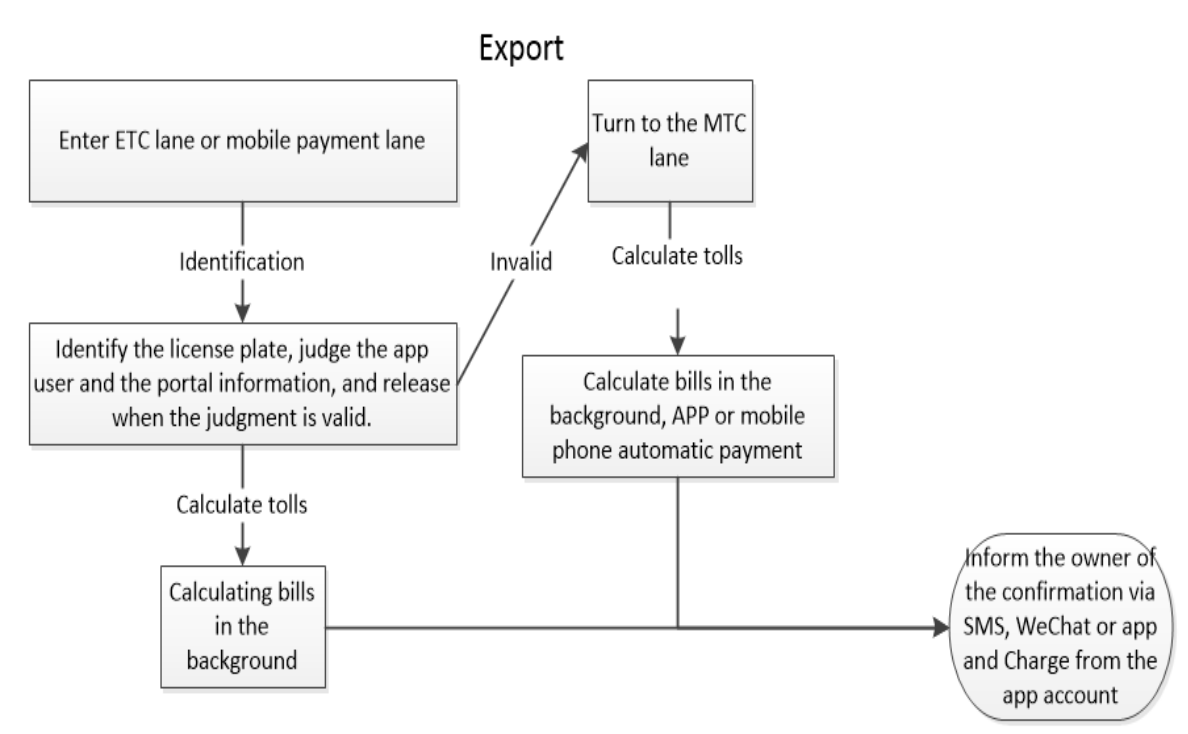

Fig. 3 Flow chart of export

\subsection{User Registration Process}

(1) Install the province's highway free parking mobile payment app.

(2) Apply for an account through the APP, and bind the license plate, model and ID card, and account. Users can pay and recharge the APP virtual account through various methods such as online banking, credit card, Alipay, WeChat, and original ETC account. When registering, the legality and matching of license plates, models and ID cards need to be confirmed in the background. If a license plate has passed the background review, it can be bound by other accounts.

\subsection{Entry Process}

(1) Add license plate recognition system in front of ETC lane or mobile payment lane.

(2) After the APP user enters the lane, the lane system identifies its license plate and model. It determines whether the APP user is valid according to the license plate, and whether the vehicle type matches.

(3) After the system recognizes that the match is successful, the lift bar is released, and the vehicle entrance information is uploaded to the cloud center. If the system recognizes the matching failure, the MTC lane is taken. At the same time, the owner gets the information of entering the toll booth by SMS, WeChat or app. If the owner finds the deck car, he can call the service hotline or use the APP to start the complaint procedure to ensure that the deck is intercepted at the exit.

(4) If the user drives someone else's car but chooses to pay by himself, he can log in to the mobile phone APP before entering the high speed, add another license plate binding, and then unbind after the high speed, the bill can be selectively paid by the owner or the user.

\subsection{Export Process}

(1) A license plate recognition camera is installed in front of the ETC lane, and the APP user enters the lane for license plate and vehicle type identification. According to the license plate, it is judged whether the APP user is valid, whether it is an illegal vehicle, a blacklist, a responded vehicle, and whether the model matches.

(2) If the network is unblocked, after identifying the license plate, the background intelligently searches for the entry information of the car according to the path recognition result, and lifts the lever release. If the network is not smooth, the system will identify the registered users of the local storage and the credit information database after identifying the license plate and vehicle type. If the credit is good, the lever will be released. The toll is calculated in the background, notified to the user via SMS or APP, and then debited from the APP account. 
(3) If system identification fails or system failure, it can be handled by portable equipment and manual remote operation. Or transfer to the manual lane, the toll collector uses the driver's mobile phone APP for non-cash charges. Or the driver informs the toll collector to automatically deduct the fee from the APP system, print invoice charges or direct deductions, etc.

(4) After the high speed, the time limit confirmation notification is sent to the owner's SMS or WeChat, mobile APP. If there is a dispute to initiate the appeal procedure, the license plate is included in the audit list, and the mobile phone non-stop payment function is not allowed before the appeal processing is completed. If there is no dispute, the system deducts the fee from the APP account, and when the balance is sufficient, the deduction is completed and the APP sends a notification. On the contrary, when the balance is insufficient, the system determines whether the bound payment method allows automatic confidential deduction. If the deduction is allowed and the balance is sufficient, the deduction is completed and the user is reminded. If not, the SMS or APP notifies the user to complete the payment within the time limit. Otherwise, it will affect the evaluation of credit information and the next high-speed trip. Within the time limit, the user completes the payment and APP notification, else it is included in the bank credit information and Internet credit information system, and is included in the high-speed blacklist. The following is the flow chart of deduction after the high speed.

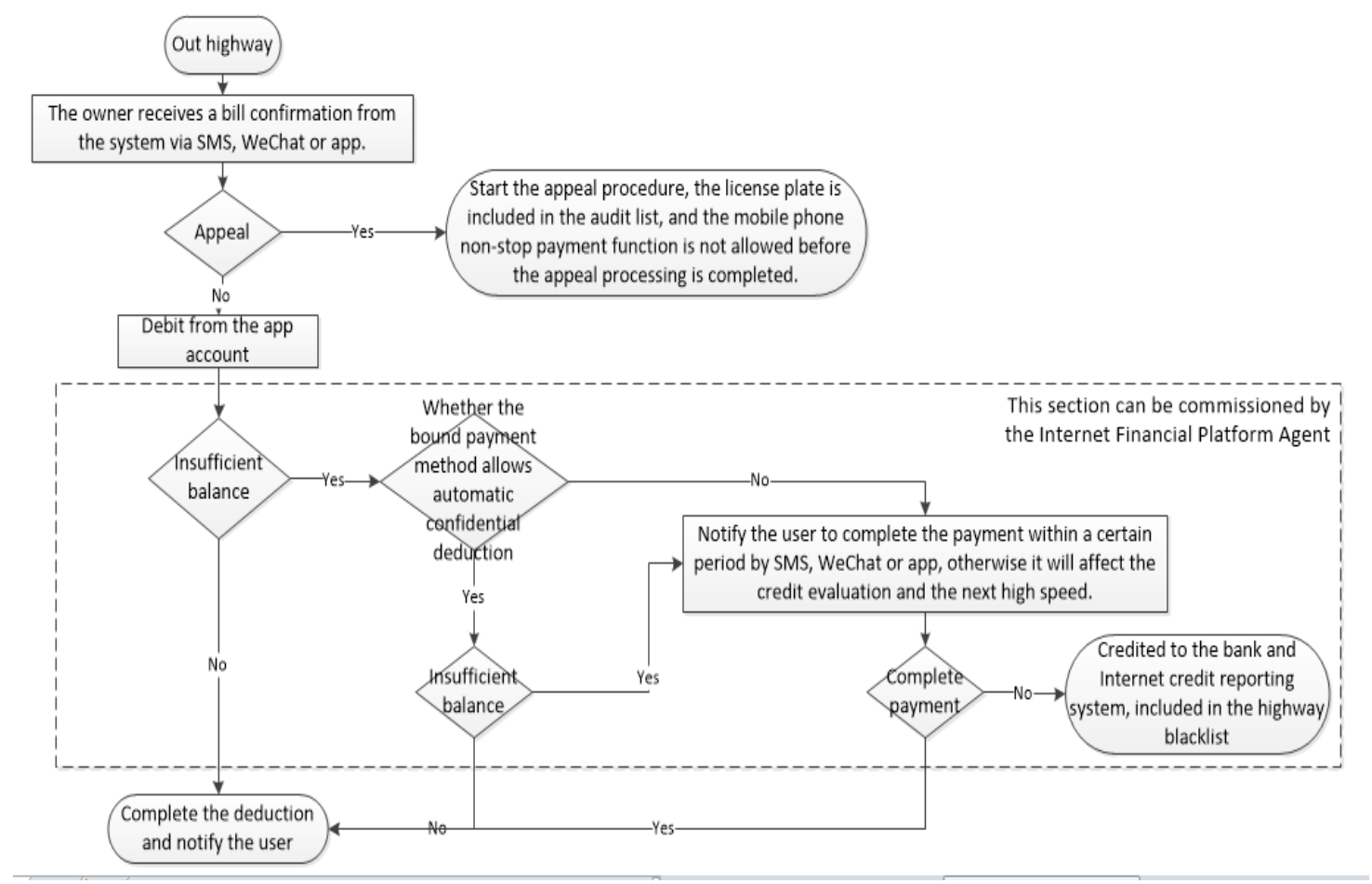

Fig. 4 Flow chart of deduction after the high speed

It is recommended to renovate two mobile phone payment lanes for one in and one out at conditional toll stations. For toll stations that do not have a dedicated lane, first deploy in the ETC lane.

At present, the ETC lane capacity is still abundant, but the MTC is basically saturated. It is better to renovate the ETC lane and attract some vehicles in the MTC lane to change the ETC lane, which will improve the toll efficiency of the toll station as a whole. The transformation of the ETC lane must be carried out steadily and must be rigorously tested to ensure that the license plate recognition rate and network security meet the design requirements. After ensuring the full compatibility with the ETC system, the ETC lane can be fully reconstructed.

\section{Application Verification}

(1) Mobile APP system: The mobile APP includes functions such as personal center, recharge and payment, SMS verification and reminder, customer service and appeal, record inquiry, etc., providing customers with one-stop high-speed traffic service; 
(2) Background service system: Including customer management subsystem, charge management subsystem, settlement management subsystem and resource management subsystem. Realize the unified management of people, finances and related to mobile APP system;

(3) Customer service system: Customer Service Center (including telephone, SMS, WeChat, APP customer complaints, consultation questions and front-end service support);

(4) Analysis and tracking system: It mainly include user behavior analysis on mobile payment methods, credit evaluation, stolen evasion tracking system, system abnormality, etc.

\section{Conclusion}

Based on the "License Plate Recognition + Mobile APP" mode, this paper achieves the goal of self-service application for non-stop, non-cash payment on the expressway without additional equipment. Based on the Internet, cloud computing and big data, this paper realizes a quick trading method without parking, no card and no cash, and this method coexists with existing ETC and MTC charging methods. It is a technology and management innovation to implement "Internet + convenient transportation" and improve the efficiency of expressway traffic. The project technical plan is advanced, intelligent, convenient and feasible.

With the evolution of charging methods, mobile payment technologies and electronic identification license plate technology, the existing multiple charging methods (such as ETC, MTC) will be over-extended to the electronic license plate recognition charging method in the future.

\section{References}

[1]. QiuYan, QinShengping, Sunxiaozhong, New scheme of intelligent tolling system for expressway, J. Computer Engineering and Design. 2004 (07): 1080-1081+1115.

[2]. LiuBinbin, Application of license plate recognition system in high speed charging, J. Image \& Multimedia Technology. 2017 (08): 75.

[3]. ZhangBo, Expressway non-stop mobile payment platform based on Internet of Things, J. China ITS Journal. 2017 (09): 96-103.

[4]. Wang Qiulan, Research on Application of Highway Toll System Based on Mobile Payment, J. China New Telecommunication. 2017, 19 (17): 85-86.

[5]. DingFengqi, Discuss about domestic highway toll mobile payment, J. China ITS Journal. 2017 (11): 94-96.

[6]. LuoQingyi, YangBo, Sun Deqiang, Application of mobile payment in the field of high-speed road toll collection, J. China ITS Journal. 2017 (11): 91-93.

[7]. Wang Guogang, LvWupian, Chen Liuchang, Application of intelligent redundant license plate recognition technology in the toll road non-stop mobile payment system, J. China ITS Journal. 2017 (12): 95-99+120.

[8]. Cao Xiaofeng, exploring the system of non-stop moving and paying for high-speed roads, J. China ITS Journal. 2018 (02): 110-112+126.

[9]. Huang Weizhong, Analysis of Expressway Non-stop Charging System Based on License Plate Recognition and Mobile Payment, J. China MUNICIPAL Engineering. 2018 (03): 27-30+116.

[10]. CuiHongjun, MaYuanzheng, LiXia, JiaJunbo. Architecture Design of New Highway Nonstop Charging System Based on Network Payment, J. Highway, 2018, 63 (05): 201-205. 